\title{
apc: An R Package for Age-Period-Cohort Analysis
}

by Bent Nielsen

\begin{abstract}
The apc package includes functions for age-period-cohort analysis based on the canonical parametrisation of Kuang et al. (2008a). The package includes functions for organizing the data, descriptive plots, a deviance table, estimation of (sub-models of) the age-period-cohort model, a plot for specification testing, plots of estimated parameters, and sub-sample analysis.
\end{abstract}

\section{Introduction}

Age-period-cohort models are extensively used in actuarial sciences, demography, epidemiology and social sciences. They have an identification problem in that the predictor is defined from time effects for age, period and cohort, but these time effects cannot be fully recovered from the predictor. The apc package, see Nielsen (2015a), implements the solution proposed by Kuang et al. (2008a) and Nielsen (2014), which is to abandon the time effects and reparametrise the predictor in terms of freely varying parameters. The vector of freely varying parameters is of a lower dimension than the vector of the original time effects. These freely varying parameters describe the variation of the likelihood function fully. The intention with the package is to focus on the aspects of the time effect that are identified by the likelihood.

The age-period-cohort model has three time scales: age, period and cohort. These are linked through the identity age + cohort $=$ period. The package is concerned with the situation where two of the time scales are measured in discrete and equidistant time. The third time scale can then be computed through age + cohort $=$ period. The choice of these two indices vary from application to application. For instance, the example in this paper is a an age-period array of annual counts of mesothelioma deaths by age group. The interface of the package is constructed in such a way that the user does not need to keep track of the coordinate system. Internally, the package uses an age-cohort coordinate system to exploit that period is a symmetric function of age and cohort.

The statistical model is a generalized linear model with a predictor of the form

$$
\mu_{\text {age, } \text { cohort }}=\alpha_{\text {age }}+\beta_{\text {period }}+\gamma_{\text {cohort }}+\delta .
$$

The likelihood is a function of the predictor $\mu_{\text {age,cohort }}$. In turn, the predictor is constructed from time effects for age, $\alpha_{\text {age }}$, period, $\beta_{\text {period }}$, and cohort, $\gamma_{\text {cohort }}$. If only we could estimate the time effects, we could learn about the predictor through manipulations of the time effects. This would be done by treating these as time series: plot them, fit time series models to them, perhaps forecast future values, and finally combine them to get the predictor. However, the time effects are not fully identifiable from the predictor so this approach has to be pursued with some care.

The identification problem is that linear trends can be moved between the time effects without changing the predictor. Indeed, the predictor in (1) satisfies

$$
\begin{aligned}
\mu_{\text {age }, \text { cohort }}=\left(\alpha_{\text {age }}+a+d \times \text { age }\right)+\left(\beta_{\text {period }}+b\right. & -d \times \text { period }) \\
& +\left(\gamma_{\text {cohort }}+c+d \times \text { cohort }\right)+(\delta-a-b-c),
\end{aligned}
$$

for any choice of $a, b, c$ and $d$. In other words, knowledge of the predictor from the likelihood is not enough to pin down the time effects. The problem is discussed, for instance, by Carstensen (2007), Clayton and Schifflers (1987a,b), Holford (1985), Kuang et al. (2008a), Luo (2013), Nielsen and Nielsen (2014), O'Brien (2011), and Yang and Land (2013). There appears to be two types of solutions to the problem: either to introduce four constraints to the time effects or to abandon the time effects and seek a parsimonious and freely varying parametrisation of the predictor. The apc package follows the latter approach. With the former approach, the constraints must come from some external argument as the likelihood carries no information in this respect. An example is the 'intrinsic estimator', which is based on a particular choice of a generalized matrix inverse, see Yang and Land (2013). The constraints need to be tracked carefully through the analysis to clearify which inferences are driven by data and which inferences are driven by the constraints; see Nielsen and Nielsen (2014) for an algebraic analysis both for frequentist and Bayesian settings.

The apc package addresses the identification through the parsimonious parametrization of the predictor suggested by Kuang et al. (2008a), see also Nielsen (2014). This exploits the fact that the second differences of the time effects are identified and that the predictor itself is also identifiable. 
As an example, the age second difference is $\Delta^{2} \alpha_{\text {age }}=\Delta \alpha_{\text {age }}-\Delta \alpha_{\text {age-1 }}$, where the first differences are $\Delta \alpha_{\text {age }}=\alpha_{\text {age }}-\alpha_{\text {age-1 }}$. The second differences are identifiable from the predictor through

$$
\Delta^{2} \alpha_{\text {age }}=\mu_{\text {age,cohort }}-\mu_{\text {age }-1, \text { cohort }+1}-\mu_{\text {age }-1, \text { cohort }}+\mu_{\text {age }-2, \text { cohort }+1} .
$$

The double differences are well-known to be identifiable in the age-period-cohort model (1), see for instance Clayton and Schifflers (1987b). Martínez Miranda et al. (2015) give a log-odds interpretation of the double differences. If the double differences are all zero, or equivalently, all time effects are linear then the model reduces to a linear plane. That linear plane is parametrised by the any three elements of the predictor, $\mu_{\text {age, cohort }}, \mu_{\text {age }}{ }^{+}$, cohort $^{\dagger}, \mu_{\text {age }}^{\ddagger}$, cohort ${ }^{\ddagger}$ say, for which the coordinates form a triangle rather than a line. Accordingly, Kuang et al. (2008a) suggest to use

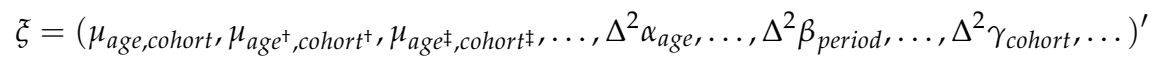

A dimension reduction of 4 is achieved, since double differencing reduces each set of time effects by two elements. The parameter is invariant to the identification problem (2) due to (3). To be of any use it has to be shown how the predictor can be formed from the parsimonious parameter. Double summation of double differences of the time effects results in the original time effects up to a linear trend. Thus, for the user of the package it is sufficient to know that predictor can be found from the parsimonious parameter through a formula of the form

$$
\mu_{\text {age,cohort }}=\text { a linear plane }+\sum_{\text {age }} \sum^{2} \alpha_{s}+\sum_{\text {period }} \sum^{2} \beta_{s}+\sum_{\text {cohort }} \sum^{2} \gamma_{s} .
$$

The formula that is actually used internally in the package is shown in (11). In that parametrisation the linear plane is a function exclusively of $\mu_{\text {age,cohort }}, \mu_{\text {age }}{ }^{\dagger}$, cohort $^{+}, \mu_{\text {age }}^{\ddagger}$, cohort so $^{\ddagger}$ so that linear plane parameters and double differences are separated. The formula that is used in default plots uses detrended versions of the double sums of double differences, see (15). This allows the user to focus on deviations from linearity. The parsimonious predictor is identified since it can be shown that different values $\xi^{\dagger} \neq \xi^{\ddagger}$ imply different predictors $\mu^{\dagger} \neq \mu^{\ddagger}$, see Kuang et al. (2008a). In the context of an exponential family $\xi$ is therefore the canonical parameter and the family is regular.

An existing package, Epi, for age-period-cohort analysis is created by Carstensen et al. (2014). It is based on Carstensen (2007). It has a series of functions for demographic and epidemiological analysis as well as some functions for age-period-cohort analysis. There are several differences between the packages apc and Epi. First, apc uses the canonical parametrization of Kuang et al. (2008a), whereas Epi does not. Second, apc, at present, is concerned with age-period-cohort data in various matrix formats. These have to be vectorized before fitting the generalized linear model, but this is done internally, so that the user only has to consider the original matrix format, while Epi takes data in vectorized form and uses the data frame format. Third, at present, apc cannot handle the problem of over-lapping cohorts: the people of age 25 in April 2015 will have been born either in 1989 or in 1990. Conversely those born in 1990 will either be 24 or 25 in April 2015. When data on all three time scales are available, cells can be sub-divided into two Lexis triangles with non-overlapping cohorts. Epi has functions for exploiting such information.

The main contributions of the apc are therefore

1. to consider data in matrix format indexed in a number of different ways;

2. to provide specification graphics illustrating the quality of the fit;

3. to estimate the model parametrised in terms of the canonical parameter $\xi$ in (4);

4. to visualize the components of the representation of the predictor $\mu$ in (5) as time series;

5 . and to do this from a range of sub-models where some of the components of $\xi$ or, correspondingly, of the time effects, are set to zero.

The remainder of the paper will illustrate this. It is envisaged to extend the package with further time series tools in the future. For reference, a theory of forecasting in the age-period-cohort model is given in Kuang et al. (2008b) and used in for instance Martínez Miranda et al. (2015).

\section{The apc package}

The apc package includes functions for organizing the data, descriptive plots, a deviance table, estimation of (sub-models of) the age-period-cohort model, a plot for specification testing, plots of estimated parameters, and sub-sample analysis. These are described in turn.

The example for this analysis is a data set for annual mesothelioma deaths in the UK taken from Martínez Miranda et al. (2015). It is thought that most mesothelioma deaths are caused by exposure to 


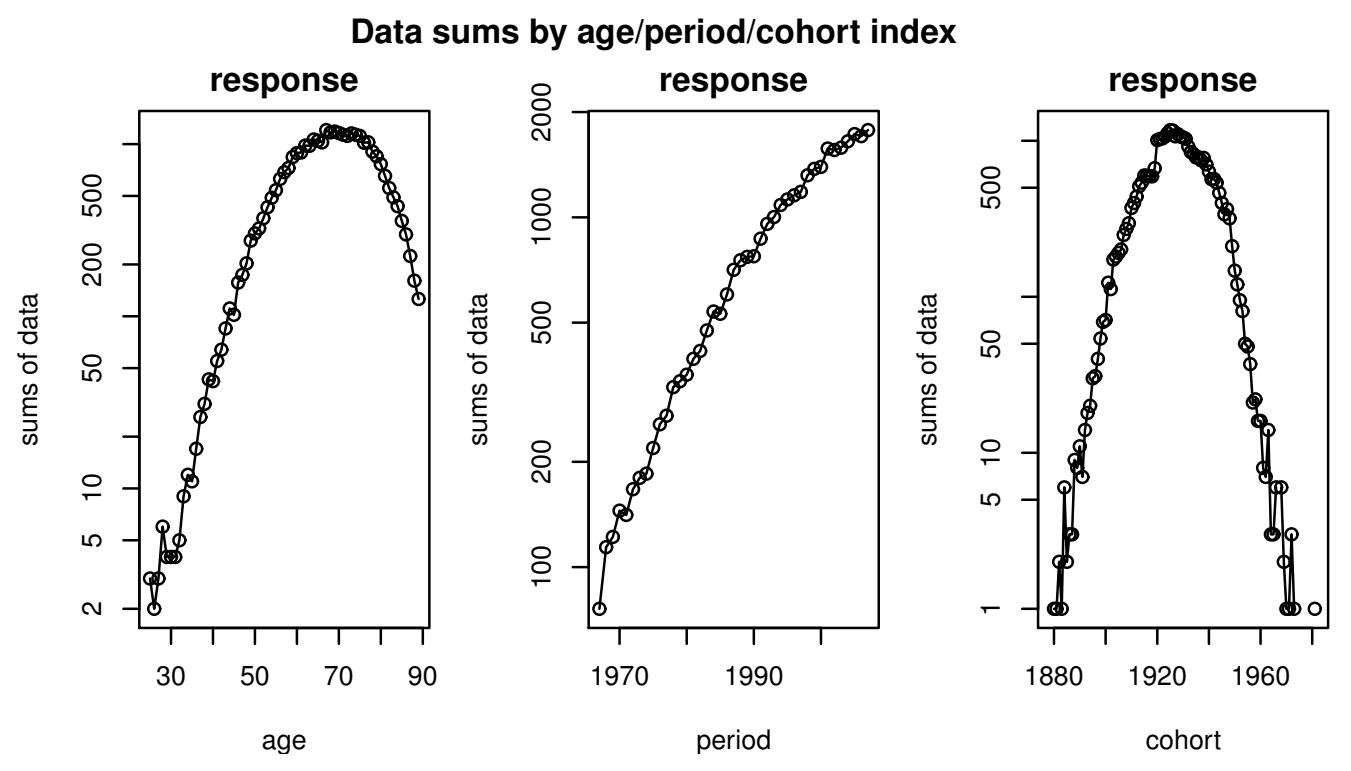

Figure 1: Data sums by age, by period and by cohort.

asbestos. The data set has counts of male deaths by age $25-89$ and by $1967-2007$. There is no direct measure for the exposure to asbestos.

\section{Organizing the data}

Age-period-cohort data may include doses and responses or just responses. They come in different types of data arrays. apc allows eight matrix formats arising from the choice of two indices from the age, period, and cohort time scales, a triangular format for chain-ladder analysis, as well as a generalized trapezoid format encompassing the other options, see (8). A special data format apc. data. list is used to keep track of the data format and the time scales. An artificial response-only data set organized in age-period format can be coded as follows

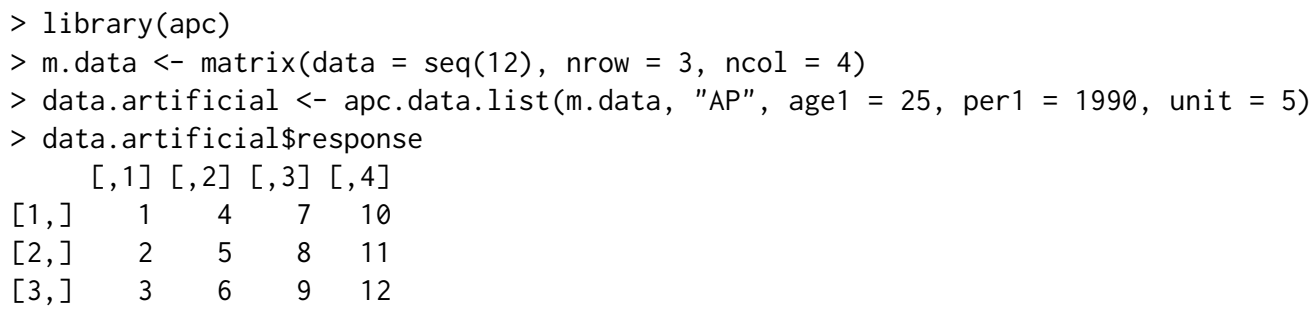

The value returned to the variable data. artificial from apc. data.list is a list with ten elements. The list includes the response, m. data; a dose which is set to NULL in this example; the data format "AP"; and information about the real time scales. This is all based on the arguments of the function apc. data. list. The first argument defines the response data, while the second argument signifies that the response matrix is rectangular with coordinates in age-period format. The remaining arguments are optional. In this case information about the times scales have been given. This shows that the real time scales are 25,30,35 for age and 1990, 1995,2000, 2005 for period, which in turn implies that the cohorts are $1955,1960, \cdots, 1980$. At this point data. artificial\$response simply stores the input matrix. We can think of it as varying in a simple age-period coordinate system. From a practical viewpoint this is not particularly helpful. Therefore apc will exploit the optional information on the real time scales when reporting estimators in the subsequent analysis.

A variety of data from the literature are pre-coded including the asbestos data from Martínez Miranda et al. (2015). The available information for that data set is exactly as in the previous example: a data matrix for responses in period-age format, though much larger, along with information about the time scales. It can be called through

$>$ data.asbestos <- data.asbestos() 


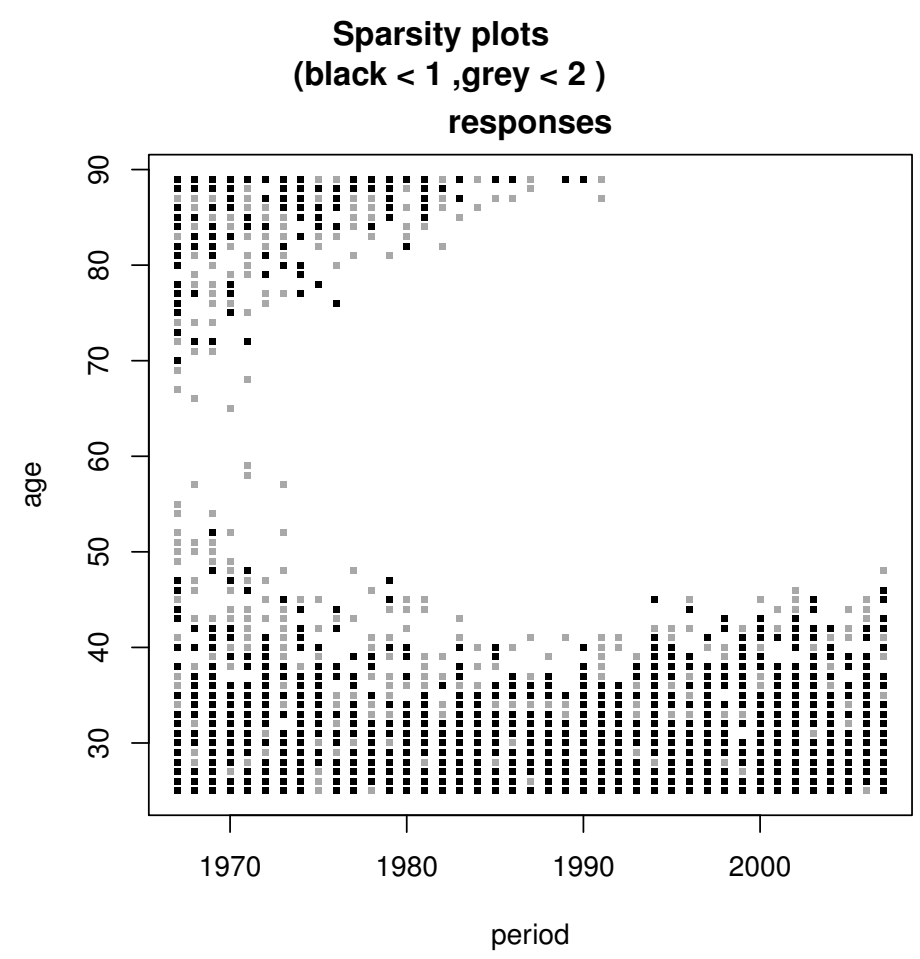

Figure 2: Sparsity plot.

\section{Descriptive plots}

The apc package has a variety of plots for descriptive analysis. These include plots of sums of the data by age, period, or cohort to get an idea of the aggregate development; plots of the data matrix against two of the three time indices to spot patterns in the data; and sparsity plots indicating if some entries in the data matrix are very small. For instance, there are very few mesothelioma deaths for young people. These plots can be called and manipulated individually or they can be called with a single command, for example:

$>$ apc.plot.data.all(data.asbestos)

Figure 1 shows the plots of data sums. The responses are seen to be sparse for young people and for old and recent cohorts. The sparsity plot in Figure 2 illustrates this in more detail. It shows with black and grey entries in the data matrix with zero or one. The data are very sparse for young age groups and for old cohorts.

\section{Deviance analysis}

At this point the distribution is chosen. Currently four distributions are implemented: A Poisson response model, a Poisson dose-response model, a logistic dose-response model, and a Gaussian model giving least squares regression. The sampling theory for the two Poisson models is described in Martínez Miranda et al. (2015) and Nielsen (2014), respectively.

The age-period-cohort model has a variety of interesting sub-models. These arise by setting some of the coordinates of the canonical parameter $\xi$ to zero. Nielsen (2014) gives a detailed discussion of the interpretation of the sub-models. An age-cohort model "AC" arises by setting the period doubledifferences to zero, so $\Delta^{2} \beta_{j}=0$ for $j=1, \ldots, J$. The drift models of Clayton and Schifflers $(1987 \mathrm{a}, \mathrm{b})$ arise by setting two sets of double-differences to zero. An age-drift model "Ad" arises by setting the double differences $\Delta^{2} \beta_{j}$ and $\Delta^{2} \gamma_{k}$ to zero. Thus, it is a sub-model of "AC". An age model code"A" arises by by setting $\Delta^{2} \beta_{j}, \Delta^{2} \gamma_{k}$, and the cohort slope to zero. Thus, it is a sub-model of "Ad". A trend model " $t$ " is the linear plane where all double differences $\Delta^{2} \alpha_{i}, \Delta^{2} \beta_{j}$ and $\Delta^{2} \gamma_{k}$ are set to zero. Thus, it is a sub-model of "Ad", but not nested in "A" as it has a cohort slope. An age trend model arises by setting all double differences $\Delta^{2} \alpha_{i}, \Delta^{2} \beta_{j}$ and $\Delta^{2} \gamma_{k}$ as well as the cohort slope to zero. Thus it is a sub-model of both " $t$ " and "Ad". Finally, an intercept model is denoted " 1 ". A deviance table gives an overview of the relative performance of the different models. For the mesothelioma data we get the 
following output.

$>$ apc.fit.table(data.asbestos, "poisson.response")

$-2 \log L$ df.residual prob(>chi_sq) LR.vs.APC df.vs.APC prob(>chi_sq) aic

$\begin{array}{lrrrrrrr}\text { APC } & 2384.923 & 2457 & 0.848 & \text { NA } & \text { NA } & \text { NA } 10805.81 \\ \text { AP } & 5336.034 & 2560 & 0.000 & 2951.111 & 103 & 0.00013550 .92 \\ \text { AC } & 2441.728 & 2496 & 0.778 & 56.805 & 39 & 0.033 & 10784.61 \\ \text { PC } & 8265.746 & 2520 & 0.000 & 5880.823 & 63 & 0.00016560 .63 \\ \text { Ad } & 5912.422 & 2599 & 0.000 & 3527.499 & 142 & 0.00014049 .31 \\ \text { Pd } & 23461.384 & 2623 & 0.000 & 21076.461 & 166 & 0.00031550 .27 \\ \text { Cd } & 8494.658 & 2559 & 0.000 & 6109.735 & 102 & 0.00016711 .54 \\ \text { A } & 21948.036 & 2600 & 0.000 & 19563.113 & 143 & 0.00030082 .92 \\ \text { P } & 34391.044 & 2624 & 0.000 & 32006.121 & 167 & 0.00042477 .93 \\ \text { C } & 28415.983 & 2560 & 0.000 & 26031.060 & 103 & 0.00036630 .87 \\ \text { t } & 24037.772 & 2662 & 0.000 & 21652.849 & 205 & 0.00032048 .66 \\ \text { tA } & 40073.386 & 2663 & 0.00037688 .463 & 206 & 0.00048082 .27 \\ \text { tP } & 34967.432 & 2663 & 0.000 & 32582.509 & 206 & 0.00042976 .32 \\ \text { tC } & 50558.531 & 2663 & 0.000 & 48173.607 & 206 & 0.00058567 .42 \\ 1 & 51003.046 & 2664 & 0.000 & 48618.123 & 207 & 0.00059009 .93\end{array}$

The first column in the table has the heading -2logL, noting that the deviance for Poisson and logistic models can be interpreted as minus twice the log likelihood for the model normalized to be zero in the saturated model. The deviance table indicates that the reduction worth considering is an age-cohort model, which is denoted "AC". Moreover, the likelihood value and $p$-value for the "APC" model indicate that the quality of the unrestricted model is quite good, with a deviance smaller than the degrees of freedom.

\section{Estimation of a particular model}

We can look a bit closer at a particular sub-model. For instance, in the case of the asbestos data the unrestricted age-period-cohort model is estimated as follows. The estimation in apc is based on the representation (11). Along with the estimates we get standard errors, which are discussed below. The canonical parameter has 208 parameters, so only the first 8 estimates are reported here.

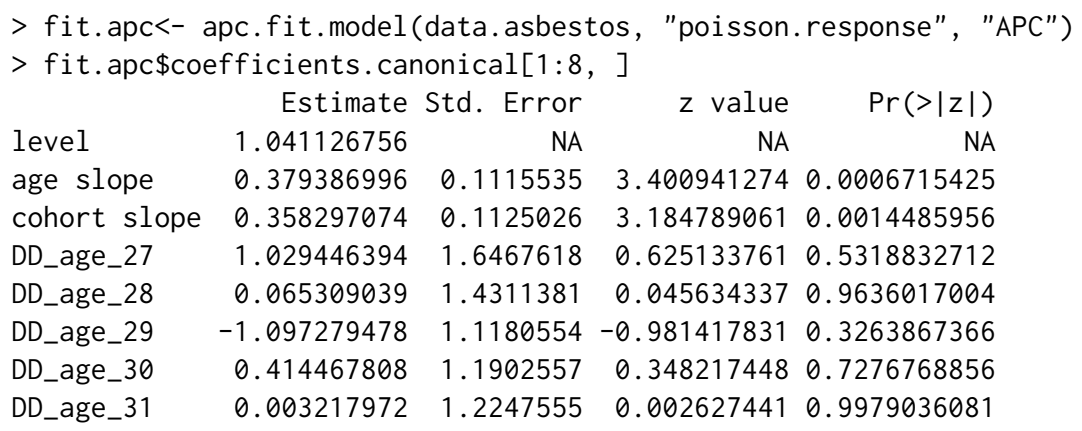

Note that the names for the parameters utilize the information about the real time scales coded through apc. data.list().

For this data set exposure or dose is not available. We therefore apply the multinomial sampling scheme used in Martínez Miranda et al. (2015). With this approach we condition on the overall level of the data. The asymptotic distribution approximations will therefore be good in a situation where the dimension of the data is fixed and the total number of responses is large. Thus, in this response model we do not get standard errors for the level.

The level and the age and period slope define the linear plane that would arise if all double differences were set to zero. The interpretation derives from the general representation (11). The level is the estimate of the predictor $\mu_{57,1967,1910}$, which is the predictor of the middle age group for the lowest period. The slopes have more interesting interpretations. The age (cohort) slope shows how much the predictor changes when increasing age (cohort) by one, while keeping cohort (age) fixed. Thus, the age and cohort slopes estimate

$$
\mu_{58,1968,1910}-\mu_{57,1967,1910,} \quad \mu_{57,1968,1911}-\mu_{57,1967,1910}
$$

where any cell could be taken as a reference point. While the level and slopes have an explicit interpretation it is perhaps easier to interpret them in terms of a plot. Plots of the estimates are discussed below. While the package parametrises the linear plane in terms of the age and cohort slopes 


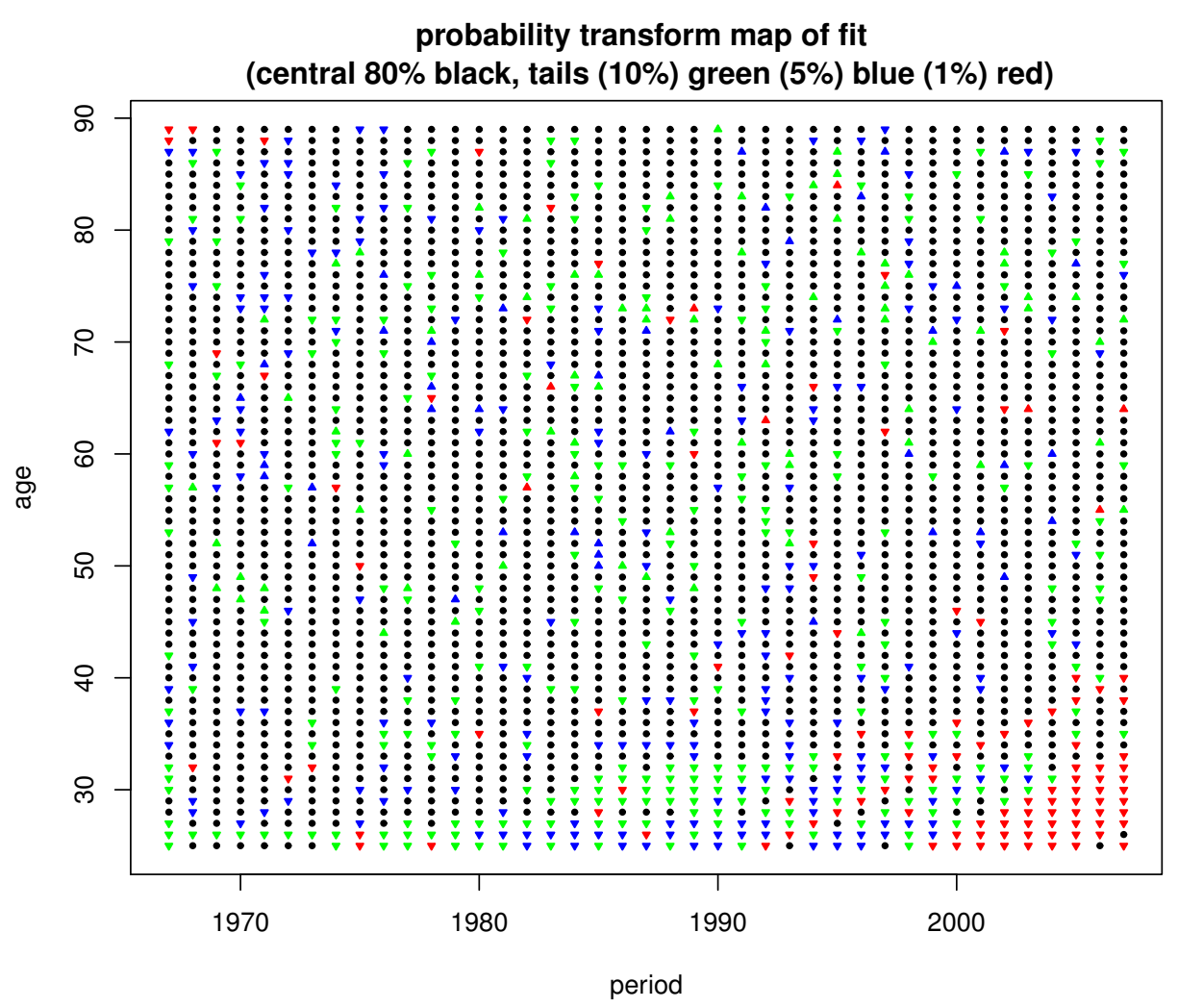

Figure 3: Probability transform plot of age-period-cohort fit to asbestos data.

other choices could be made, such as age and period slopes. The age and cohort slopes are chosen due to the age and cohort symmetry of the model.

\section{A probability transform plot for the fit}

The quality of the fit can be illustrated using a probability transform plot. Using the estimates it plots probability transforms of responses given the fitted value. In other words: are the actual observations probable given the estimated model? The plot is given in the original coordinate system. Colours and symbols are used to indicate whether responses are central to the fitted distribution or in the tails of the fitted distribution. The intention of the plot is to reveal if there are particularly many extreme observations given the fit and if they form a particular pattern.

For the asbestos data the probability transform plot is coded as:

$>$ apc.plot.fit.pt(fit.apc)

Figure 3 shows the result. For instance, all red point triangles indicates observations in the extreme 1 $\%$ of the distribution. Those pointing down indicate the lower end of the distribution. The number of red triangles is not particular large given the number of observations, $n=2665$, but, they form a pattern among the most recent cohorts. Therefore, a sub-sample analysis is performed below.

\section{Plots of the estimates}

The estimates can be plotted using a single command. This command will automatically pick up information about which sub-model and adjust accordingly, based on the analysis in Nielsen (2014). There are two types of plots, which are illustrated using a sequence of three plots. Details follow.

1. Figure 4. Plot of type "sum. sum". This is illustrates the canonical parameter and the representation (11), but it is possibly the less useful choice in practical work.

2. Figure 5. Plot of type "detrend". This is illustrates the representation (15). It is the default choice.

3. Figure 6. Plot of type "detrend" for a sub-sample. The above plots appear very messy, in part because of the sparsity. This evidence leads to a sub-sample, for which the estimates look much cleaner. 


\section{APC canonical parameters \& standard representation model.design= APC (1/2 std blue/red)}

(a) $\Delta^{2} \alpha$

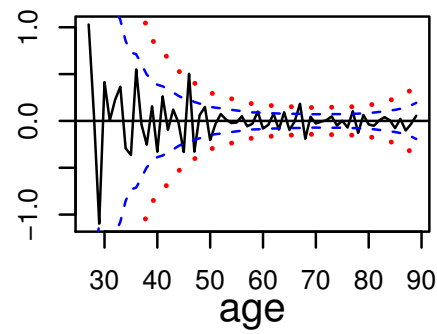

(d) first linear trend

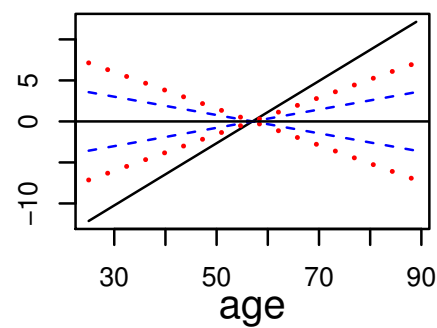

(g) $\Sigma^{2} \Delta^{2} \alpha$

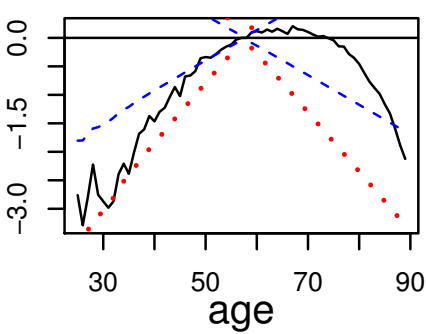

(b) $\Delta^{2} \beta$

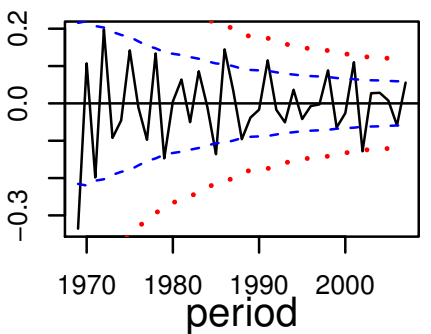

(e) aggregate mean

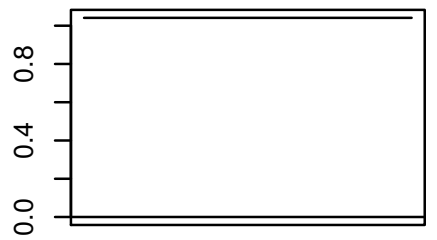

(h) $\Sigma^{2} \Delta^{2} \beta$

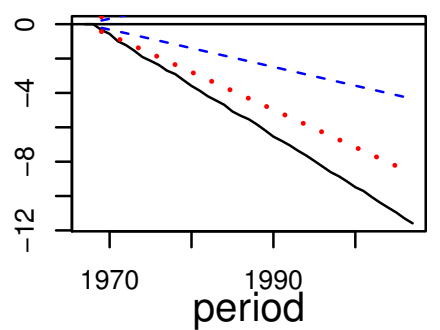

(c) $\Delta^{2} \gamma$

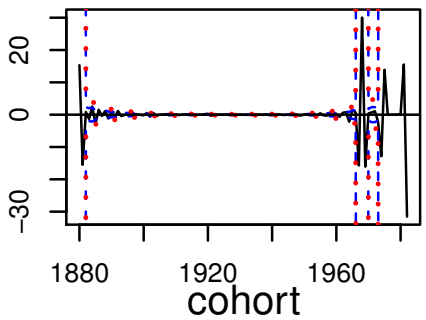

(f) second linear trend

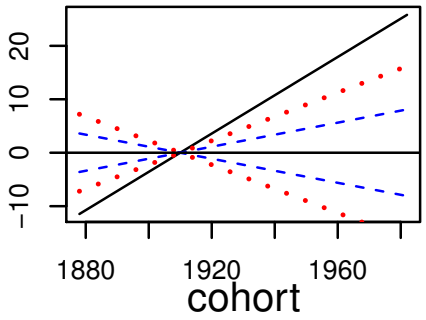

(i) $\Sigma^{2} \Delta^{2} \gamma$

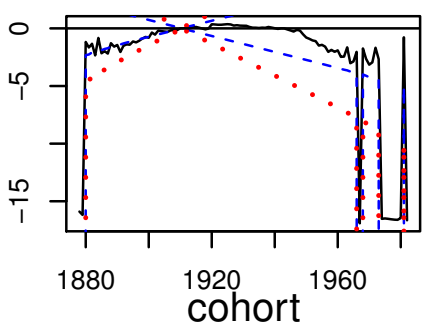

Figure 4: Plots of the fitted canonical parameters illustrating representation (11).

Plot of type "sum. sum". Figure 4 is generated by:

$>$ apc.plot.fit(fit.apc, type $=$ "sum.sum")

It shows the canonical parameter estimates and illustrates the representation (11).

Figure 4 (a)-(c) shows the estimated second difference parameters $\Delta^{2} \alpha_{i}, \Delta^{2} \beta_{j}, \Delta^{2} \gamma_{k}$. The estimates are plotted with pointwise confidence bands centered around zero. The age double differences are noisy for young ages while the cohort double differences are noisy for young and old cohorts. This is due to the sparsity of observations for those age and cohort groups as shown in Figure 2. This calls for a sub-sample analysis, which is described below.

The next row of panels in Figure 4 illustrates the estimated level and the slopes (6). Panel (e) shows the estimated level of 1.04. No confidence bands are shown due to the multinomial sampling scheme. Panels (d), (f) show the age and cohort slopes anchored at age 57 and cohort 1910 as discussed above.

Figure 4(g)-(i) shows double sums of double difference based on the representation (11). In each plot two values of the double sums are set to zero. In other words, the degrees of freedom, that is the number of non-zero values, in these plots are exactly the same as for the double differences. For exact values of the double sums see the last section of the paper.

The sum of the information in Figure 4(d)-(i) gives the linear predictor of the model. That, is for someone born in 1920 and dying at age 70 in 1990, the predictor is the sum of the linear age trend in (d) evaluated at 70, the level in (e), the linear cohort trend in (f) evaluated at 1920, the age effect in (g) evaluated at 70, the period effect in (h) evaluated at 1990, and the cohort effect in (i) evaluated at 1920.

The plots have a messy appearance. There are several reasons. First, double sums of double differences are only identified up to arbitrary linear trends. It can be difficult to abstract from that arbitrary linear trend with these plots. In particular the period effect in (h) has a strong linear trend. It is hard to discern what the variation around that linear trend could be. We address this in the 


\section{APC canonical parameters \& detrended representation model.design= APC (1/2 std blue/red)}

(a) $\Delta^{2} \alpha$

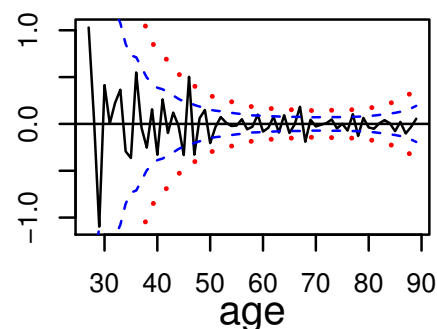

(d) first linear trend

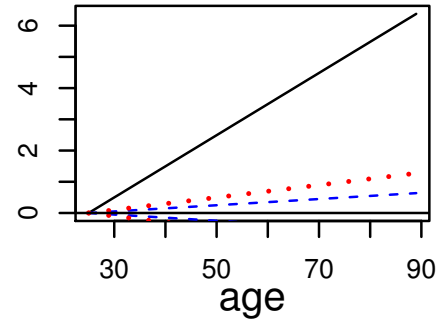

(g) detrended $\Sigma^{2} \Delta^{2} \alpha$

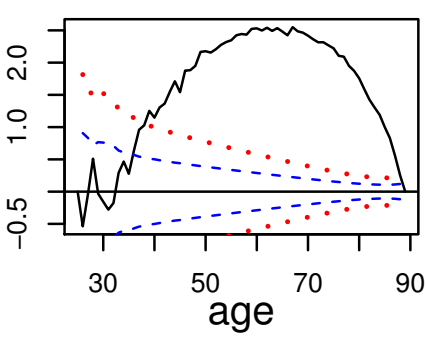

(b) $\Delta^{2} \beta$

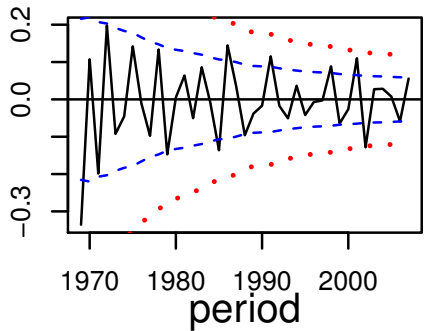

(e) aggregate mean

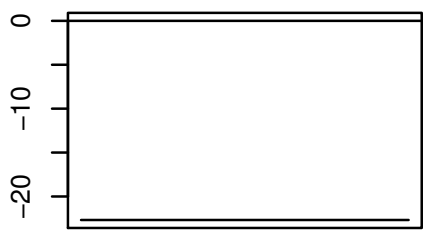

(h) detrended $\Sigma^{2} \Delta^{2} \beta$

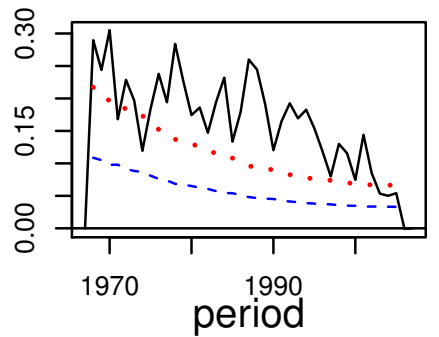

(c) $\Delta^{2} \gamma$

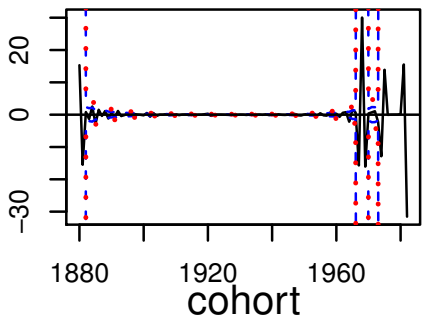

(f) second linear trend

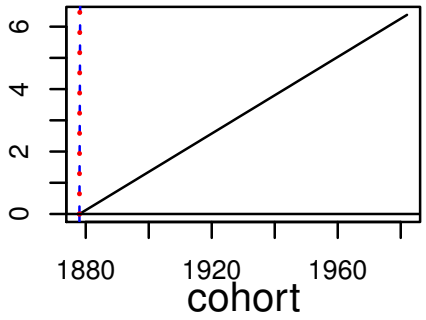

(i) detrended $\Sigma^{2} \Delta^{2} \gamma$

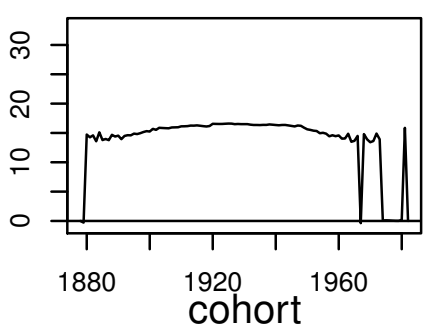

Figure 5: Plots of the fitted values using the detrended representation (15).

sub-sample analysis in connection with Figure 5. Second, the data are sparse for young age groups and for young and old cohorts. This shows up in panels (a),(c),(g),(i). We address this in the sub-sample analysis in connection with Figure 6.

Plot of type "detrend". Figure 5 is generated by:

$>$ apc.plot.fit(fit.apc)

This plot illustrates the detrended representation (15). Figure 5(a)-(c) show exactly the same double differences as before.

The level, slopes and double sums in Figure 5(d)-(i) are now changed. The idea is to give a good visual impression of variation over and above a linear trend while preserving the degrees of freedom in panels (a)-(c). In this way Figure 5(g)-(i) show double sums of double differences detrended so as to start and end in zero. The level and slopes in Figure 5(d)-(f) are then changed according to representation (15). The interpretation is as before: The linear predictor for someone born in 1920 and dying at age 70 in 1990 is the sum of the linear age trend in (d) evaluated at 70, the level in (e), the linear cohort trend in (f) evaluated at 1920, the detrended age effect in (g) evaluated at 70, the detrended period effect in (h) evaluated at 1990, and the detrended cohort effect in (i) evaluated at 1920.

There are several noteworthy features of the detrended plots. The detrended double sums in Figure 5(g)-(i) fill the plot area better than those in Figure 4(g)-(i). Visually, it is easier to abstract from the arbitrary linear trend and focus on deviation from the linear trend. A possible drawback of the detrended plot is that age-period-cohort models can have perfect fit in some corners of the data array. For an age-period array, the very first and last cohort double differences will therefore be based on one data entry each. When looking at the detrended double sums those double differences are, however, 


\section{APC canonical parameters \& detrended representation model.design= APC (1/2 std blue/red)}

(a) $\Delta^{2} \alpha$

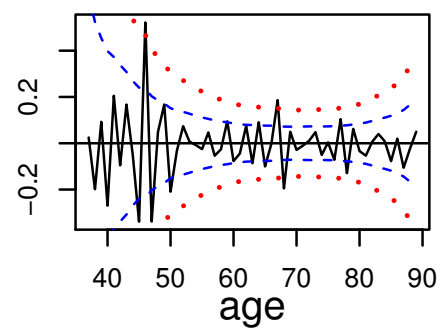

(d) first linear trend

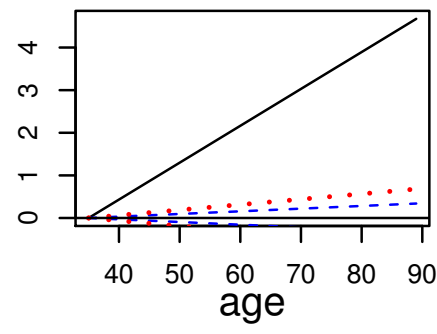

(g) detrended $\Sigma^{2} \Delta^{2} \alpha$

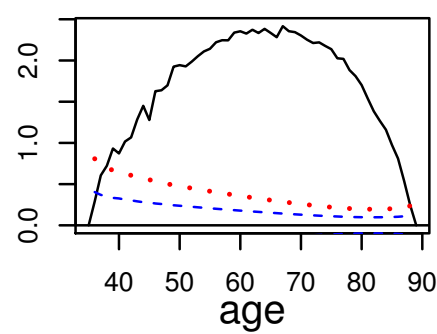

(b) $\Delta^{2} \beta$

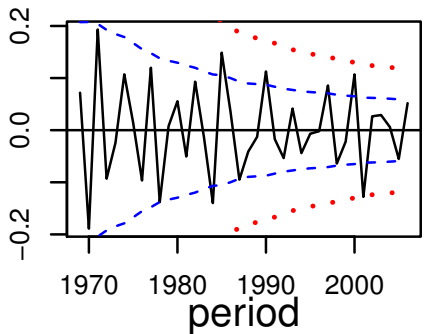

(e) aggregate mean

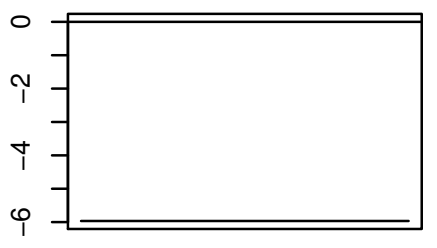

(h) detrended $\Sigma^{2} \Delta^{2} \beta$

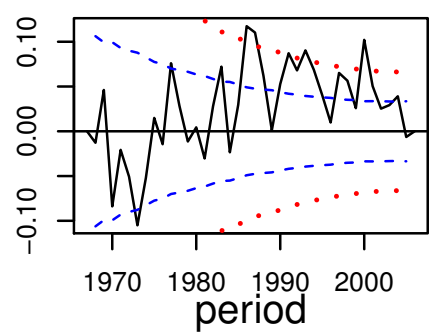

(c) $\Delta^{2} \gamma$

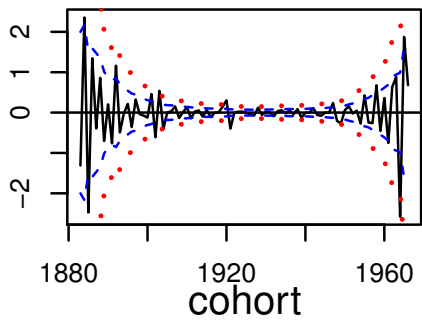

(f) second linear trend

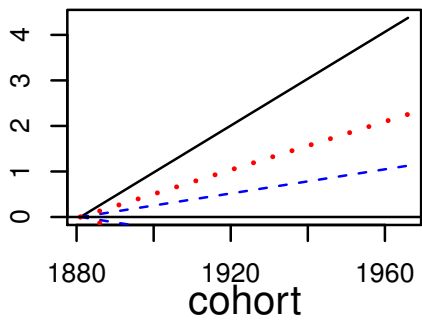

(i) detrended $\Sigma^{2} \Delta^{2} \gamma$

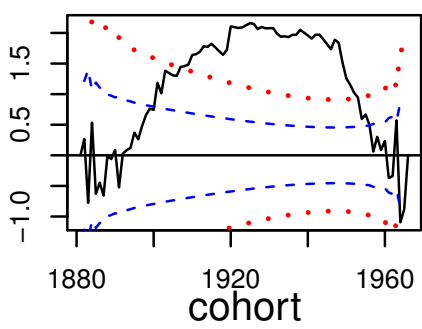

Figure 6: Plots of the fitted values for sub-sample.

combined with double sums of all the other double differences which are better determined.

The detrended age double sums in Figure 5(g) are broadly similar to those in Figure 4(g) apart from a lift in the scale and a slight change of slope. The plot indicates a near concave deviation from linearity after age 35 . The development over the range 25-35 could be driven by the sparsity of the data in that region. We return to this point in the sub-sample analysis.

The detrended period double sums in Figure 5(h) have a very different appearance from those in Figure 4(h). The appearance is now seen to be a ragged concave shape. The first period stands out. Abstracting from that, the plot looks very linear. We return to this point in the sub-sample analysis.

The detrended cohort double sums in Figure 5(h) are just as messed up in appearance as those in Figure 4(h). The confidence bands have dropped off the plot and a warning is given. Again, the sub-sample analysis will address this point.

Plot of type "detrend" for a sub-sample. Figure 6 shows the result of a sub-sample analysis.

The asbestos data is sparse for low ages and for old and young cohorts. A recursive analysis can be used to check how sensitive the above analysis is in this respect. The idea is to cut parts of observations away and redo the analysis. This can be done through the command:

$>$ data.asbestos.subset <- apc.data.list.subset(data.asbestos, $10,0,0,0,3,16$ )

which cuts the lower 10 age groups, the lower 3 cohort groups and the upper 16 groups. The subset of the data is no longer a rectangle in the period-age coordinate system, but rather a rectangle with some corners cut off. This is a generalized trapezoid, see (8) for details. The above analysis can now be redone. The deviance table, which is not reported, gives approximately the same information as before, with only weak support for the "AC" sub-model $(p=4 \%)$.

Prompted by the jump in the period effect in Figure 5(h) we can go one step further and drop the 
data for the first period, 1967. It is possible that the data collection scheme was slightly different the first year. This is also consistent with Tan et al. (2010). We achieve this with only a small modification of the previous code

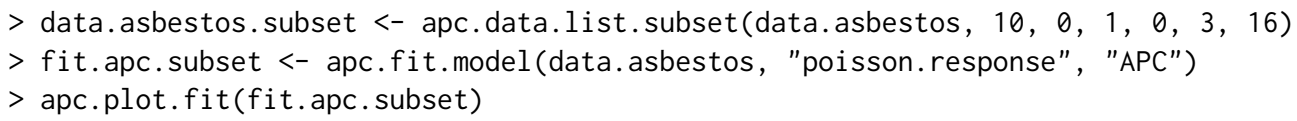

The deviance table, which is not reported, now gives stronger support for the "AC" sub-model ( $p=$ $12 \%)$.

Figure 6 shows plots of the estimates. The difference relative to Figure 5 is that the noise from the youngest age groups, the first period group and the youngest and oldest cohorts group has been eliminated. Remarkably, the estimates for the remaining age, period and cohort groups are very similar. This is most clear in the plot of $\Delta^{2} \beta$ in panels (b) of Figure 5,6, which are nearly identical. The plots of $\Delta^{2} \alpha$ and $\Delta^{2} \gamma$ in panels (a), (c) are also very similar, although this is masked by the difference in scales. For a good empirical model the predictors for the sub-sample should be the same in the full sample and in the sub-sample. Since the double differences are identifiable from the predictors, the same should apply to them.

The double sums of double differences in panels (g)-(i) and the consequent level and linear slopes in (d)-(f) are changed. They depend on the normalisation, which depends on the choice of sample. For the sub-sample, we now see a concave shape in the double sums for age and cohort. Since the sums are pinned down to be zero at both ends this is very visible. This is quite common in cancer studies. Nielsen (2014) argues that this is consistent with double differences that increase from a negative value and sub log linear age effects.

It is worth noting that the sub-sample analysis is used in two ways here. First, it is used to trim off noise from sparse parts of the data set. Secondly, it is used to show that estimates do not depend very much on the choice of data array. Martínez Miranda et al. (2015) are concerned with forecasting future mortality and use the sub-sample analysis to show that forecasts are robust to the choice of data array. However, at present forecast methods are not implemented in apc. For an identification theory of forecasting see Kuang et al. (2008b). Recursive sub-sample graphs are very common in time series econometrics, see Hendry and Nielsen $(2007, \S 13.4)$ and could, with advantage, be developed further.

\section{Some details on the representation}

Internally apc uses a representation developed in Nielsen (2014) that generalises the representation (5) from Kuang et al. (2008a). An overview of the representation is given along with some notes on the level and slope estimates in the mesothelioma example as well as on the ad hoc identification of double sums of double differences and the application to the mesothelioma data.

\section{The representation}

The package can handle data arrays that are generalized trapezoids. To illustrate this, while keeping a notation that is consistent with Nielsen (2014) the age-period-cohort model (1) is now written as

$$
\mu_{i k}=\alpha_{i}+\beta_{j}+\gamma_{k}+\delta,
$$

where $i$ is age, $j$ is period and $k$ is cohort, so that $i+k=j+1$. The generalized trapezoids are arrays of the form

$$
\mathcal{I}=\{i, k: 1 \leq i \leq I, 1 \leq k \leq K, L+1 \leq j \leq L+J\},
$$

where $I, J$ and $K$ are the numbers of age, period and cohort indices, while $L+1$ is the lower period index. An age-cohort rectangular array arises when $L=0$ and $J=I+K-1$. A reserving triangle is a triangular age-cohort array where $I=J=K, L=0$. A period-age rectangular array is an age-cohort trapezoid where $L=I-1$ and $K=I+J-1$. The above sub-sample analysis is based on a rectangular age-cohort array with two corners chopped off.

It is convenient to choose a representation of the model that is symmetric in age and cohort. Nielsen (2014) derives such a representation. The level is anchored in the middle of the first diagonal of odd length. Thus, define $U=\operatorname{integer}\{(L+3) / 2\}$. For a period-age array where $L=I-1$ this reduces to $U=\operatorname{integer}(I+2) / 2$. If $I$ is odd the anchoring point will be the middle age group $(I+1) / 2$ for the first period. If $I$ is even the anchoring point will be the age group $I / 2+1$ for the second period. The age and cohort slopes are then define as the one-step slopes in age and cohort directions from that point. The canonical parameter is then chosen as

$$
\xi=\left(v_{0}, v_{a}, v_{c}, \Delta^{2} \alpha_{3}, \ldots, \Delta^{2} \alpha_{I}, \Delta^{2} \beta_{L+2}, \ldots, \Delta^{2} \beta_{L+J}, \Delta^{2} \gamma_{3}, \ldots, \Delta^{2} \gamma_{K}\right)^{\prime},
$$


where

$$
v_{0}=\mu_{U U}, \quad v_{a}=(i-U)\left(\mu_{U+1, U}-\mu_{U U}\right), \quad v_{c}=(k-U)\left(\mu_{U, U+1}-\mu_{U U}\right) .
$$

It can then be shown that the predictor has the representation

$$
\mu_{i k}=v_{0}+v_{a}+v_{c}+A_{i}+B_{j}+C_{k}
$$

where

$$
\begin{aligned}
A_{i} & =1_{(i<U)} \sum_{t=i+2}^{U+1} \sum_{s=t}^{U+1} \Delta^{2} \alpha_{s}+1_{(i>U+1)} \sum_{t=U+2}^{i} \sum_{s=U+2}^{t} \Delta^{2} \alpha_{s} \\
B_{j} & =1_{(L \text { odd } \& j=2 U-2)} \Delta^{2} \beta_{2 U}+1_{(j>2 U)} \sum_{t=2 U+1}^{j} \sum_{s=2 U+1}^{t} \Delta^{2} \beta_{s} \\
C_{k} & =1_{(k<U)} \sum_{t=k+2}^{U+1} \sum_{s=t}^{U+1} \Delta^{2} \gamma_{s}+1_{(k>U+1)} \sum_{t=U+2}^{k} \sum_{s=U+2}^{t} \Delta^{2} \gamma_{s}
\end{aligned}
$$

Estimates for the time effects $A_{i}, B_{j}, C_{k}$ can be found by the code

$>$ id.apc <- apc.identify(fit.apc)

$>$ id.apc\$coefficients.ssdd

The canonical parameter (9) and the predictor (11) can be visualized through the command

$>$ apc.plot.fit(fit.apc, "sum.sum")

The interpretation is similar to that given in the discussion of Figure 5 . The package includes a vignette, Nielsen (2015b), showing how the parameters $A_{i}, B_{j}, C_{k}$ are computed from the canonical parameter.

The representation (11) has the advantage that it is symmetric in age and cohort, reducing to that of Kuang et al. (2008a) for age-cohort data arrays. There is some separation between the linear plane parameters and the non-linear parameters. Indeed, the transformation from (9) to (11) does not mix the two. The choice of parametrisation is primarily for internal uses and will usually not be of importance to the user. It should be noted that any bijective transformation of $\xi$ could be used as the parsimonious parameter. If the transformation is linear, the transformed parameter will also be the canonical parameter in an exponential family context. Some times non-linear transformations of the parameter are preferred. An example is the chain ladder model, which is an age-cohort model for an age-cohort triangle. This is often parametrised in terms of the development factors, see Kuang et al. (2009) for a discussion.

\section{The detrended representation}

The default plot of the parameters uses a detrended version of the parameters $A_{i}, B_{j}, C_{k}$. To be specific, it uses the representation

$$
\mu_{i k}=v_{0}^{\text {detrend }}+(i-1) v_{a}^{\text {detrend }}+(k-1) v_{c}^{\text {detrend }}+A_{i}^{\text {detrend }}+B_{j}^{\text {detrend }}+C_{k}^{\text {detrend }},
$$

where

$$
\begin{aligned}
& A_{i}^{\text {detrend }}=A_{i}-A_{1}-\frac{i-1}{I-1}\left(A_{I}-A_{1}\right) \\
& B_{j}^{\text {detrend }}=B_{j}-B_{1}-\frac{j-L-1}{J-1}\left(B_{L+J}-B_{L+1}\right) \\
& C_{k}^{\text {detrend }}=C_{k}-C_{1}-\frac{k-1}{K-1}\left(C_{K}-C_{1}\right),
\end{aligned}
$$

which all start and end in zero. Consequently, it must hold that

$$
\begin{aligned}
v_{0}^{\text {detrend }} & =v_{0}-(U-1)\left(v_{a}+v_{c}\right)-A_{1}-B_{J+1}-C_{1}-\frac{L}{J-1}\left(B_{L+J}-B_{L+1}\right), \\
v_{a}^{\text {detrend }} & =v_{a}+\frac{1}{I-1}\left(A_{I}-A_{1}\right)+\frac{1}{J-1}\left(B_{J+L}-B_{J+1}\right), \\
v_{c}^{\text {detrend }} & =v_{c}+\frac{1}{K-1}\left(C_{K}-C_{1}\right)+\frac{1}{J-1}\left(B_{J+L}-B_{J+1}\right) .
\end{aligned}
$$

The parameters $v_{0}^{\text {detrend }}, v_{a}^{\text {detrend }}, v_{c}^{\text {detrend }}, A_{i}^{\text {detrend }}, B_{j}^{\text {detrend }}, C_{k}^{\text {detrend }}$ can be found by the code 
$>$ fit.apc\$coefficients.detrend

They are visualized in Figures 5. In particular, the plotted level and slopes are those derived in (19)(21). The vignette Nielsen (2015b) shows how to check the transformation from the representation (11) to $(15)$.

\section{The level and slope estimates for the mesothelioma data}

Recall that the canonical parameter estimates for the mesothelioma data are available through:

\begin{tabular}{|c|c|c|c|c|}
\hline & Estimate & Std. Error & z value & $\operatorname{Pr}(>|z|)$ \\
\hline level & 1.041126756 & NA & NA & NA \\
\hline age slope & 0.379386996 & 0.1115535 & 3.400941274 & 0.0006715425 \\
\hline ohort slope & 0.358297074 & 0.1125026 & 3.184789061 & 0.0014485956 \\
\hline DD_age_27 & 1.029446394 & 1.6467618 & 0.625133761 & 0.5318832712 \\
\hline D_age_28 & 0.065309039 & 1.4311381 & 0.045634337 & 0.9636017004 \\
\hline
\end{tabular}

The level estimate for the mesothelioma arises as follows. The data is organised in an periodage array with $I=65$ age groups and $J=41$ cohort groups. Thus, $L=I-1=64$ and $U=$ integer $\{(L+3) / 2\}=$ integer $(67 / 2)=33$. The anchoring point for the level is therefore in the agecohort coordinate system $\mu_{33,33}$, or, in an period-age coordinates $\mu_{1,33}$. The corresponding, real time period-age coordinates are $\mu_{1967,57}$. To check this predictor estimates the level, run the following code, which organises the linear predictor for the vectorized data as a matrix in the original format.

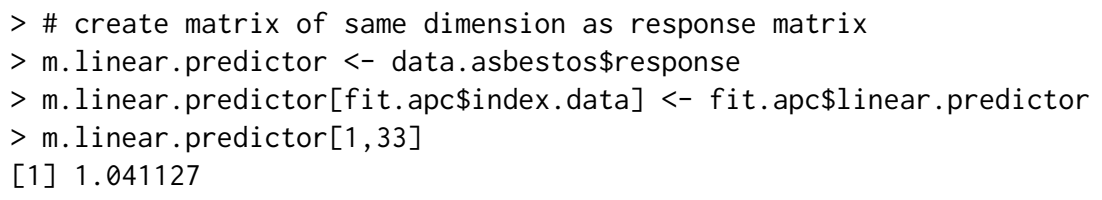

For comparison, there are 5 observed deaths of age 57 in 1967, which is not far from $\exp \left(\widehat{\mu}_{1967,57}\right)=2.8$.

The slope estimates arise as follows. The age and cohort slopes are now, in age-cohort coordinates, $\mu_{34,33}-\mu_{33,33}$ and $\mu_{33,34}-\mu_{33,33}$, or, in period-age coordinates, $\mu_{2,34}-\mu_{1,33}$ and $\mu_{2,33}-\mu_{1,33}$. The estimates are

$>$ m.linear.predictor $[2,34]-m$. linear.predictor $[1,33]$

[1] 0.379387

$>$ m.linear.predictor $[2,33]-m$. linear.predictor $[1,33]$

[1] 0.3582971

The estimates of the double sums appearing in (11) can be computed by as follows.

\begin{tabular}{|c|c|c|c|c|}
\hline & & Std. Error & z value & $\operatorname{Pr}(>|z|)$ \\
\hline SS_DD_age_56 & -0.02995345 & 0.09120714 & -0.3284113 & 0.7426007 \\
\hline _DD_age_57 & 0.00000000 & NA & NA & NA \\
\hline -OD_dge_s & 0.00000000 & NA & NA & NA \\
\hline 9 & 0.09970809 & 0.08965228 & 1.1121646 & 0.2660674 \\
\hline _1967 & 0.00000000 & NA & NA & NA \\
\hline eriod_1968 & 0.00000000 & NA & NA & NA \\
\hline eriod_1969 & -0.33556503 & 0.21566380 & -1.5559636 & 7167 \\
\hline 39 & -0.15147783 & 0.12248429 & -1.2367124 & 0.2161939 \\
\hline & 0.00000000 & NA & NA & NA \\
\hline _DD_cohort_1911 & 0.00000000 & NA & NA & NA \\
\hline _DD_cohort_1912 & 0.02337873 & 0.12074650 & 0.1936183 & 0.8464748 \\
\hline
\end{tabular}

\section{Summary}

This article describes the apc package for age-period-cohort modelling. It implements the canonical parametrisation of Kuang et al. (2008a). The package includes functions for organizing the data, a descriptive plot, a deviance table, estimation of sub-models of the age-period-cohort model, a plot for specification testing, plots of estimated parameters, and sub-sample analysis. 


\section{Acknowledgements}

Comments from an anonymous referee and from B. Carstensen are gratefully acknowledged.

\section{Bibliography}

B. Carstensen. Age-period-cohort models for the Lexis diagram. Statistics in Medicine, 26(15):3018-3045, 2007. [p52, 53]

B. Carstensen, M. Plummer, E. Laara, and M. Hills. Epi: A Package for Statistical Analysis in Epidemiology, 2014. URL https://CRAN.R-project.org/package=Epi. R package version 1.1.67. [p53]

D. Clayton and E. Schifflers. Models for temporal variation in cancer rates. I: Age-period and agecohort models. Statistics in Medicine, 6(4):449-467, 1987a. [p52, 55]

D. Clayton and E. Schifflers. Models for temporal variation in cancer rates. II: Age-period-cohort models. Statistics in Medicine, 6(4):469-481, 1987b. [p52, 53, 55]

D. F. Hendry and B. Nielsen. Econometric Modeling. Princeton University Press, 2007. [p61]

T. R. Holford. An alternative approach to statistical age-period-cohort analysis. Journal of Chronic Diseases, 38(10):831-836, 1985. [p52]

D. Kuang, B. Nielsen, and J. P. Nielsen. Identification of the age-period-cohort model and the extended chain ladder model. Biometrika, 95(4):979-986, 2008a. [p52, 53, 61, 62, 63]

D. Kuang, B. Nielsen, and J. P. Nielsen. Forecasting with the age-period-cohort model and the extended chain-ladder model. Biometrika, 95(4):987-991, 2008b. [p53, 61]

D. Kuang, B. Nielsen, and J. P. Nielsen. Chain-ladder as maximum likelihood revisited. Annals of Actuarial Science, 4(1):105-121, 2009. [p62]

L. Luo. Assessing validity and application scope of the intrinsic estimator approach of the age-periodcohort problem. Demography, 50(6):1945-1967, 2013. [p52]

M. D. Martínez Miranda, B. Nielsen, and J. P. Nielsen. Inference and forecasting in the age-periodcohort model with unknown exposure with an application to mesothelioma mortality. Journal of the Royal Statistical Society A, 178(1):29-55, 2015. [p53, 54, 55, 56, 61]

B. Nielsen. Deviance analysis of age-period-cohort models. Nuffield College Discussion Paper, 2014. [p52, 55, 57, 61]

B. Nielsen. apc: A Package for Age-Period-Cohort Analysis, 2015a. URL https: //CRAN. R-project.org/ package=apc. R package version 1.1. [p52]

B. Nielsen. Checking the identification of the apc package, 2015b. URL https: //CRAN. R-project.org/ package=apc. Vignette for the apc package. $[p 62,63]$

B. Nielsen and J. P. Nielsen. Identification and forecasting in mortality models. The Scientific World Journal, pages 1-24, 2014. Article ID 347073. [p52]

R. M. O'Brien. Constrained estimators and age-period-cohort models. Sociological Methods E Research, 40(3):419-452, 2011. [p52]

E. Tan, N. Warran, A. J. Darnton, and J. T. Hodgson. Projection of mesothelioma mortality in Britain using Bayesian methods. British Journal of Cancer, 103(3):430-436, 2010. [p61]

Y. Yang and K. C. Land. Age-Period-Cohort Analysis: New Models, Methods, and Empirical Applications. Chapman \& Hall/CRC, Boca Raton, FL, 2013. [p52]

Bent Nielsen

Nuffield College, Oxford OX1 1NF, UK

and

Department of Economics, University of Oxford

and

Programme for Economic Modelling, INET, Oxford

bent.nielsen@nuffield.ox.ac.uk 